\title{
SOS

\section{A Social Network Analysis Approach to Diagnosing and Improving the Functioning of Transdisciplinary Teams in Public Health}

\author{
Sarah Gehlert, $P h D^{1,2}$ Bobbi J. Carothers, $P h D^{3}$ Jung Ae Lee, $P h D^{5}$ Jefferson Gill, $P h D^{4}$ \\ Douglas Luke, $P h D^{1,3}$ Graham Colditz, MD, PhD $D^{2,5}$ \\ ${ }^{1}$ The George Warren Brown School of Social Work, Washington University, St. Louis, MO, USA, Email: sgehlert@wustl.edu; \\ ${ }^{2}$ The Siteman Cancer Center, Washington University, St. Louis, MO, USA ${ }^{3}$ Center for Public Health Systems Science, \\ Washington University, St. Louis, MO, USA; ${ }^{4}$ Department of Political Science, Washington University, St. Louis, MO, USA; \\ ${ }^{5}$ Division of Public Health Sciences, Department of Surgery, Washington University, St. Louis, MO, USA
}

doi: $10.22545 / 2015 / 00070$

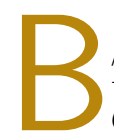
ackground: The National Cancer Institute's Transdisciplinary Research in Energetics and Cancer initiative is in its second round of funding. Despite increasing agreement that transdisciplinary team-based research is valuable in addressing complex problems like energy balance and cancer, methods for constructing and maintaining transdisciplinary teams is lacking.

Purpose: We articulate a method for assessing transdisciplinary teams that relies on social network analysis and using this knowledge to improve their functioning.

Methods: Using data from the Washington University TREC site in 2011 and 2013, we demonstrate the use of social network analysis to assess and provide feedback on team functioning.

Results: We portray broker functioning in both years. By 2013, the director and co-director had begun to share broker functions with other members. Some brokers fostered communication with less central net- work members.

Conclusions: The information obtained can help to train a new generation of investigators to optimally participate on transdisciplinary research teams.

Keywords: transdisciplinary research team, social network analysis, transdisciplinary assessment.

\section{Introduction}

Transdisciplinary research gained attention in medicine in the 1990s and early 2000s, when the National Cancer Institute mandated use of the approach in funding initiatives aimed at addressing tobacco use, disparities, and energy balance in cancer. The Transdisciplinary Tobacco Use Research Centers, Centers for Population Health and Health Disparities, and Transdisciplinary Research in Energetics and Cancer were funded in 1997, 2003, and 2005, respectively. The impetus for their development was a desire to capture the complexity of phenomena 
Sarah Gehlert, Bobbi J. Carothers, Jung Ae Lee, Jefferson Gill, Douglas Luke, Graham Colditz

A Social Network Analysis Approach to Diagnosing and Improving the Functioning of Transdisciplinary Teams in Public Health

like cancer disparities using teams of disciplinary scholars that spanned the social, behavioral, and biological sciences to provide an integrated, holistic approach.

Transdisciplinary research differs from multidisciplinary and interdisciplinary research in the extent to which investigators operate outside the boundaries of their own disciplines to share language, pool knowledge and theories, and develop new methods of analysis. In multidisciplinary research, investigators come together to solve a research problem, but approach it through separate disciplinary lenses. They might, for example, gather at the beginning of a research project with separate but related research questions, collect and analyze data independently, form independent conclusions based upon their separate research questions, and come together at the end of the project to try to make sense of it all. Very rarely do conclusions derived from such a multidisciplinary approach fit together neatly into a coherent whole, and investigators exit the collaboration with no discernible change in their approaches to science. Investigators working interdisciplinarily transfer disciplinary knowledge to one another for the purposes of research, and may share research questions, yet resurrect their disciplinary boundaries when an answer has been found that serves the needs of their root disciplines.

Transdisciplinary research, which Rosenfield defines as exchanging information, altering disciplinespecific approaches, sharing resources, and integrating disciplines to achieve a common scientific goal [1], represents the highest degree of disciplinary collaboration. Hall et al. describe its four phases as: (1) development, in which a transdisciplinary team is assembled; (2) conceptualization, during which research questions are refined; (3) implementation, which involves negotiating shared models and goals; and, (4) translation, through which discoveries are translated into change [2]. In this approach, disciplinary scholars transcend and operate outside their own boundaries to achieve synergy, mutually-inform one another's work, and create a new intellectual space in which no one discipline dominates and no way of knowing is privileged over others. The approach has the potential to forge new understandings of major public health problems by breaking down the usual barriers to shared scholarship. Emmons et al. use energetics and cancer as an example of the tremendous inefficiency that occurs when bound- aries between the social, behavioral, and biological are rigidly maintained in research, saying "If the primary focus of work in obesity and energy balance is on sociocultural factors, eventually the limits of not considering both environmental and physiologic factors will be realized [3, p. S205]."

\section{The Transdisciplinary Research on Energetics and Cancer Initiative}

The mission of the National Cancer Institute's Transdisciplinary Research on Energetics and Cancer (TREC) initiative, which includes multiple sites around the country, is to foster collaboration across multiple disciplines and projects to cover the spectrum from the biology, genetics, and genomics of energy balance to social and behavioral influences on physical activity and nutrition, weight, energetic, and cancer risk [4]. This collaboration is meant to occur within and across TREC sites. Currently in its second five-year round of funding, the four TREC sites are the University of Pennsylvania, Washington University, the University of California-San Diego, and Harvard University, with a Coordination Center at the Fred Hutchinson Cancer Research Center in Seattle. The TRECs also have a mission to train new and established investigators to carry out integrative research on energetics and energy balance and cancer.

Each TREC has approximately four research projects and a number of cores to support their work. In addition, the initiative funds a number of within- and cross-TREC developmental research projects that are selected from applications solicited once a year by the TREC Steering Committee, which is made up of the principal and co-principal investigators of the TREC sites and the Coordination Center. The developmental projects are meant to extend the research of the TRECs into new areas of discovery and translation.

\section{Evaluating Effectiveness within and Across TREC Sites}

Considering the group of TREC investigators as a social network, or a social entity made up of a number of actors, allows the group's functioning to be 
Sarah Gehlert, Bobbi J. Carothers, Jung Ae Lee, Jefferson Gill, Douglas Luke, Graham Colditz

A Social Network Analysis Approach to Diagnosing and Improving the Functioning of Transdisciplinary Teams in Public Health

analyzed in its entirety as well as the dyadic relationships between its members. The assumption is that stronger and more frequent communication and the formation of new cross-disciplinary ties will better foster advances in the science of energy balance and cancer. It is assumed that investigators will at minimum be aware of the mission of their TREC site and of the broad objectives of its research projects. Optimally, they would be conversant in one another's work through exposure during regularly-scheduled team meetings and through the co-mentoring of trainees [5]. At the Washington University TREC site, for example, postdoctoral fellows select three mentors that span disciplines from a menu of faculty who have agreed to participate in the training program.

Investigators might communicate directly with one another, as in the case of project and core leaders, or through members of the team who serve as brokers of information about the TREC site as a whole and its shared research agenda. Brokers are defined as network members who link to otherwise unconnected members of the network [6]. They play important roles in linking members by serving as go-betweens in terms of information transfer and day-to-day communication of research activities and findings.

Brokers might be the principal mode of communication for investigators who are less centrally involved in projects and cores. Inevitably, some researchers are less involved than others, especially those whose academic responsibilities are less-well covered by TREC funding. A challenge of transdisciplinary sites is to engage investigators who are less central to the TREC funding mechanism and those who might be dispersed due to off-site clinical duties. Bringing less well-integrated investigators into communication with the team as a whole ensures the maximization of TREC's scientific discovery and translation. One example of how this might occur is when a broker is able to convey information and ideas about potential cross-site or cross-project developmental projects to other members of the network.

More ties between investigators signify greater communication within a network. The principal way of evaluating this quality is by measuring the density of social network ties, defined as the number of actual ties between network members compared to the number of potential ties. Denser networks suggest faster propagation of information and greater group cohesion [6]. Also, individuals who conduct more information tend to be more productive in terms of research goals and objectives [7].

Despite increasing agreement that transdisciplinary research is a valuable approach to addressing complex problems like energy balance and cancer, methods for constructing and maintaining effective transdisciplinary teams are lacking. In the present article, we articulate a method for diagnosing transdisciplinary teams' strengths and weaknesses that relies on social network analysis, and using this knowledge to improve their functioning. With data from the Washington University Transdisciplinary Center for Energetics and Cancer at two time points (2011 and 2013), we demonstrate how social network analysis was used to assess and provide actionable feedback on the strengths and weaknesses of the team. We then discuss how the information obtained might be used to train a new generation of investigators to optimally participate on transdisciplinary research teams.

\section{Methods}

\subsection{Study Sample}

Participants in the study were investigators involved in the TREC research site at Washington University in St. Louis, including postdoctoral trainees. Here we report on 24, 31, and 31 investigators from Washington University who were involved in the social network analysis research project in 2011, 2013 and 2014, respectively.

A list of investigators was developed by the TREC Steering Committee, and after receiving IRB approval, each was sent a letter inviting them to participate along with a copy of the social network survey. The survey was sent during the first months of the second round of funding for the TREC sites, for the purpose of establishing a baseline measure of ties. None of the sites funded during the first round of TREC funding was refunded, thus all sites were new to TREC. The survey will be re-sent yearly throughout the five years of funding to assess the increase in density of social network ties over time. In the current paper, we report on data from the first three years of data collection, using data from the Washington University site only. Note that the number of respondents for the survey is less than the network size, or the total members in the network (see Table 
Sarah Gehlert, Bobbi J. Carothers, Jung Ae Lee, Jefferson Gill, Douglas Luke, Graham Colditz A Social Network Analysis Approach to Diagnosing and Improving the Functioning of Transdisciplinary Teams in Public

Table 1: Response Rate and Network Size for the Washington University TREC site in 2011, 2013 and 2014

\begin{tabular}{lccc}
\hline & $\mathbf{2 0 1 1}$ & $\mathbf{2 0 1 3}$ & $\mathbf{2 0 1 4}$ \\
\hline Number of invited & $\mathbf{2 5}$ & $\mathbf{2 5}$ & $\mathbf{2 2}$ \\
\hline Number of respondents & $20(80 \%)$ & $20(80 \%)$ & $19(86 \%)$ \\
Professor & 9 & 9 & 8 \\
Associate Professor & 1 & 4 & 3 \\
Assistant Professor & 6 & 5 & 5 \\
Research Associate & 2 & 1 & 1 \\
Other & 2 & 1 & 2 \\
\hline Network size (N) & 24 & 31 & 31 \\
\hline
\end{tabular}

Table 2: Nature of Collaboration for the Washington University TREC site in 2011, 2013 and 2014 (unit: \% of the total ties in each year)

\begin{tabular}{ccccccc}
\hline Year & Grant & $\begin{array}{c}\text { Co-authored } \\
\text { Publication }\end{array}$ & $\begin{array}{c}\text { Co-authored } \\
\text { Presentation }\end{array}$ & Mentorship & Committee & Others \\
\hline 2011 & 37.66 & 18.18 & 11.69 & 31.17 & 35.71 & 3.9 \\
2013 & 62.5 & 28.8 & 17.93 & 30.43 & 36.96 & 5.98 \\
2014 & 43.53 & 42.94 & 28.82 & 38.82 & 38.82 & 7.06 \\
\hline
\end{tabular}

1). The number of survey respondents, who are the primary actors in the network, is 20,20 , and 19 in 2011, 2013, and 2014, respectively. The difference between the respondents and the total network actors is due to the secondary actors who are included in the network because they were designated by respondents as a link but did not themselves respond to the survey. Thus we do not know about the relationship among the secondary actors. We address this because the network density can be sensitive to the number of respondents rather than the network size, which often makes it hard to fairly compare the longitudinal social network data due to different set of respondents each time point.

\section{Measures}

\subsection{Collaboration Network}

The survey included the names of all TREC investigators and asked respondents if they currently worked with, or had worked with prior to TREC, any inves- tigators on the list in any of six situations:

1. on a study or grant;

2. on a co-authored publication;

3. on a co-authored presentation;

4. in mentoring or training;

5. on a committee or work group; or,

6 . in any other activity.

For the purposes of the present study, participants were considered to have worked with another investigator if they indicated that they currently were collaborating on any of the six listed activities. The percent of collaboration types is summarized in Table 2.

\subsection{Discipline}

Investigators were asked to choose from a list of 37 academic disciplines the one which best characterized the primary disciplinary perspective of their 
Sarah Gehlert, Bobbi J. Carothers, Jung Ae Lee, Jefferson Gill, Douglas Luke, Graham Colditz A Social Network Analysis Approach to Diagnosing and Improving the Functioning of Transdisciplinary Teams in Public

Table 3: Number of Investigators by Disciplines for the Washington University TREC site in 2011, 2013 and 2014

\begin{tabular}{lccc}
\hline Categories of Disciplines & 2011 & 2013 & 2014 \\
\hline 2. Statistics \& System & 4 & 4 & 4 \\
3. Social/Behavioral Science & 5 & 6 & 6 \\
4. Epidemiology & 4 & 5 & 5 \\
6. Medicine & 8 & 10 & 10 \\
7. Nutrition/Metabolism & 1 & 3 & 3 \\
8. Public Health Practice & 2 & 3 & 3 \\
\hline Total & 24 & 31 & 31 \\
\hline
\end{tabular}

Table 4: Network Density for the Washington University TREC site in 2011, 2013 and 2014

\begin{tabular}{lcccccc}
\hline & $\begin{array}{c}\text { Network } \\
\text { size }\end{array}$ & $\begin{array}{c}\text { No. } \\
\text { Respondents }\end{array}$ & $\begin{array}{c}\text { All } \\
\text { possible edges }\end{array}$ & $\begin{array}{c}\text { No. } \\
\text { edges }\end{array}$ & $\begin{array}{c}\text { No. } \\
\text { triangles }\end{array}$ & Density \\
\hline 2011 & 24 & 20 & 276 & 72 & 79 & 0.26 \\
2013 & 31 & 20 & 465 & 109 & 158 & 0.23 \\
2014 & 31 & 19 & 465 & 96 & 119 & 0.21 \\
$2014 \mathrm{~b}$ & 31 & 23 & 465 & 104 & 148 & 0.22 \\
\hline
\end{tabular}

work. For purposes of analysis, these responses were collapsed into eight disciplines (see Table 3).

\subsection{Density}

Density is the proportion of ties that exist among all possible ties (also called edges). From Table 4, we can see the density remains the same or slightly decreases over time. It is worth noting, however, that the density measure is sensitive to the growth of network size and the change of respondents. As a network gets larger, the density tends to decrease since the number of possible edges is $\frac{n^{2}-n}{2}$ and this grows faster proportionally than the number of relationships. Also, losing one member, especially if that person is an active collaborator may result in losing all of that member's related ties, thus reducing overall density. For this reason, it is prudent to look at the absolute number of edges in the network (Table 4). We see the network expanded the most in 2013, with 109 ties compared to 72 ties in 2011 and 96 ties in 2014. The active collaboration in 2013 is partly because there was considerable grant related work in 2013 (Table 2). Although the overall density decreased in 2014 compared to previous years (Table 4), the collaborative work represented by the number of co-authored publications and presentations increased in 2014 (Table 2). In addition, we attempted to recover 2014's missing data for the respondents who responded the survey in 2013 but did not response in 2014 by assuming that the network relations of these members remained the same through 2013-14. We add this missing imputation result in 2014b in Table 4 . The improved number of edges in 2013 and 2014 (2014b) compared to 2011 indicates general improvement of collaborative functions within the Washington University TREC site. We further investigate how the characteristics of network transform over time.

\section{Analysis}

Social network analysis was used as the principal mode of analysis in the present study. We considered the investigators of the Washington University TREC site who participated in our survey as the 


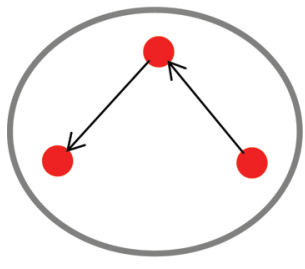

(1) Coordinator

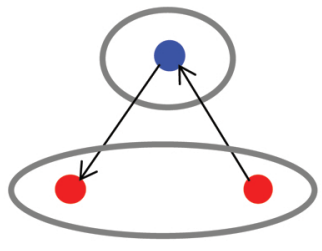

(2) Consultant

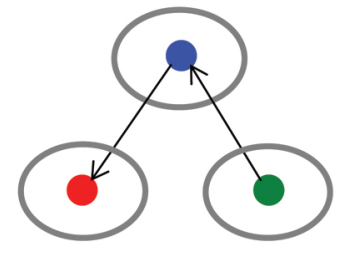

(3) Liaison

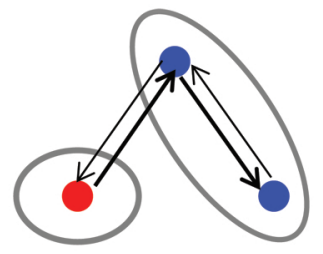

(4) Representative $(\longleftarrow)$ (5) Gatekeeper $(\longrightarrow)$

Figure 1: Illustration of the Five Brokerage Types in Directed Network. Colored dots are actors where the top point is the broker. Ellipses are the discipline boundaries.

network actors in our analyses. We define brokers as individuals who collaborate with two other network members who do not collaborate with one another, and assume mutual rather than unidimensional collaboration.

Gould and Fernandez [8] define brokerage relations based on ordered triples of actors in a sequence of communication. A coordinator broker connects two members of the same discipline of which the broker is also a member, while a consultant broker connects two members from the same discipline of which the broker is not a member (see Figure 1). A liaison broker connects two network members from different disciplines and is not a member of either of those disciplines. A representative broker connects a member of the broker's own discipline to a network member from another discipline. The gatekeeper broker involves the same three types of members (i.e., the broker and one member each from the same and a different discipline) as in a representative brokerage, but differs in the direction of information flow. The gatekeeper broker controls the flow of information from the outside-discipline member to the member of the gatekeeper's own discipline. In other words, the gatekeeper broker decides whether to grant access to a member from his own discipline, which effectively would cut that member off from network information. Because a representative and a gatekeeper broker are indistinguishable from one another in a mutual network $[6,9]$, we merged them into one category, which we designated as representative/gatekeeper.

For any ordered triple of actors $i, j$ and $k$, the situation in which $j$ is a broker between $i$ and $k$ is denoted by the symbol $i j k$. In other words, $i j k$ means $i$ is tied directly to $j, j$ is tied directly to $k$, and $i$ is not tied directly to $k$. Then, an actor $j$ 's total brokerage activity in a network with $\mathrm{N}$ actors is defined by measuring the number of ordered pairs $(i, k)$ in the network for which the condition $i j k$ holds. We modify the brokerage measure by a set of subgroups. The first type is denoted by $m_{i}=m_{j}=$ $m_{k}$ since all three actors belong to the same group. Similarly, the second type of relationship is described by $m_{i}=m_{k} \neq m_{j}$ showing that two endpoints $(i, k)$ belong to the same group while the broker $j$ is an outsider, and so forth in other types. Then, an actor $j$ 's coordinator brokerage score, named as $w_{I j}$, is defined as follows:

$$
w_{I j}=\sum_{i}^{N} \sum_{k}^{N} w_{I}(i k), \quad(i \neq j \neq k),
$$

where $\mathrm{N}$ is the number of actors in the network, and $w_{I}(i k)$ equals 1 if $i j k$ is true and if $m_{i}=m_{j}=m_{k}$ , and 0 otherwise. The other subtypes of brokerage can be computed following the same principle and named as $w_{O j}, b_{I O j}, b_{O I j}, b_{o j}$, corresponding to consultant, representative, gatekeeper, and liaison, respectively. The notation $w$ is used for the coordinator and consultant brokerage score denoting within group roles because two endpoints $(i, k)$ belong to the same subgroup. On the other hand, the representative, gatekeeper, and liaison brokerage scores are notated by $b$ because these are between group brokerage roles with two endpoints belonging to the different subgroups. Consequently, any actor $j$ 's total raw brokerage score $t_{j}$ can be calculated by the summation of the raw scores from these five subtypes. This raw measure is useful if the researcher is interested in the number of brokerage relations that an actor is capable of mediating. On the other hand, when the central issue is the degree to which the actor actually controls brokerage relations in the 
Sarah Gehlert, Bobbi J. Carothers, Jung Ae Lee, Jefferson Gill, Douglas Luke, Graham Colditz A Social Network Analysis Approach to Diagnosing and Improving the Functioning of Transdisciplinary Teams in Public Health

Table 5: Brokers by a Criterion that Standardized Brokerage Scores $>1.64$ for the Washington University TREC site in 2011, 2013 and 2014

\begin{tabular}{lccc}
\hline & \multicolumn{3}{c}{ Investigator Id } \\
\cline { 2 - 4 } Brokerage Relations & $2011(\mathrm{~N}=24)$ & $2013(\mathrm{~N}=31)$ & $2014(\mathrm{~N}=31)$ \\
\hline 1. Coordinator & $17,21,23$ & $1,5,6,17,21,31$ & $5,17,21,31$ \\
2. Consultant & 17,21 & $17,20,21$ & $17,21,28$ \\
3. Liaison & 17,21 & 17,21 & $17,21,28$ \\
4. Representative/Gatekeeper & 17,21 & $1,17,21,31$ & $1,17,21,31$ \\
\hline
\end{tabular}

network, then the appropriate measure is the partial score $t_{j}^{*}$, which can be computed analogous to $t_{j}$ but divided by $g_{i k}$. For example,

$$
w_{I j}^{*}=\sum_{i}^{N} \sum_{k}^{N} w_{I}(i k) / g_{i k}, \quad\left(i \neq j \neq k, \quad g_{i k} \neq 0\right),
$$

In which $g_{i k}$ is the number of two-step paths between $i$ and $k$. Our analysis used $t_{j}^{*}$ because our interest lies in the degree of each actor's contribution as a broker in the network rather than his or her capacity as a broker. The network as a whole can be characterized in the same terms. This is referred to as a global brokerage measure of the network and defined as

$$
W_{I}=\sum_{j}^{N} W_{I j} .
$$

We computed a standardized brokerage score for each Washington University site network member, which enables us to directly compare brokerage scores across the years [6]. The standardized scores $(\beta)$ are computed by $\beta=\left(b-\mu_{b}\right) / \sigma_{b}$, where $b$ is any of the brokerage scores defined above, $\mu_{b}$ is the expected value of $b$ and $\sigma_{b}$ is the standard deviation of $b$ under the null model. It is reasonably assumed that the standardized brokerage scores of actors follow the standard normal distribution for sufficiently large networks (about 15 actors for global scores, 30 for individual scores) [8]. Thus a higher positive score for an actor means that the actor occupies more brokerage positions than actors with lower brokerage scores. A negative extreme score, however, means that the actor avoids being a mediator in the network or prefers to operate independently. We used a 0.1 significance level, which further helped us to determine whether or not a network member was a broker. Our criterion for deciding if a network member was a broker was whether the investigator's standardized brokerage score was greater than 1.64, the approximate value of the 95 th percentile point of the standard normal distribution. The brokerage scores were calculated in the same way for each type of brokerage. We used the R package 'sna' [10] to derive functions for all brokerage scores.

In principle, we reject the null hypothesis of a random network when we observe too many or small number of actors at extremes $(>1.64$ or $<-1.64)$. This is done by comparing the number of members whose scores fall at the ends of the distribution to what we would expect in a random network. For example, in a completely random network with 31 actors, only 5 percent of the actors (1.6 actors) should have a high positive brokerage score $(>1.64)$.

\section{Results}

We observed more Washington University TREC site network members in 2013 and 2014 with brokerage scores that were higher than 1.64 (i.e., higher than expected), which led us to reject the null hypothesis for 2013 and 2014 (see Table 5). We could not, however, reject the null hypothesis for 2011. We took this to mean that while the Washington University TREC network was not particularly brokerage-oriented in 2011, it had become so by 2013 and 2014. This observation is consistent with the global measure in Table 6 . This table displays global measures of brokerage for all brokerage types and for brokerage in general. The 2013 total score is statistically significant at the 0.05 level (score > 1.96). By 2013, pairs of actors were more likely to be brokered than they were in 2011 or than they would be in a random network. 
Sarah Gehlert, Bobbi J. Carothers, Jung Ae Lee, Jefferson Gill, Douglas Luke, Graham Colditz A Social Network Analysis Approach to Diagnosing and Improving the Functioning of Transdisciplinary Teams in Public Health

Table 6: Global Brokerage Measures for the Washington University TREC site in 2011, 2013 and 2014

\begin{tabular}{lccc}
\hline & \multicolumn{3}{c}{$\begin{array}{c}\text { Standardized Global } \\
\text { Brokerage Score }\end{array}$} \\
\cline { 2 - 4 } Brokerage Relations & 2011 & 2013 & 2014 \\
\hline 1. Coordinator & 1.46 & 1.57 & 1.35 \\
2. Consultant & -0.71 & 0.76 & 0.13 \\
3. Liaison & 1.1 & 1.85 & 0.45 \\
4. Representative/Gatekeeper & 1.23 & 1.58 & 2.28 \\
\hline Total & 1.26 & 2.2 & 1.49 \\
\hline
\end{tabular}

Table 7: Standardized Brokerage Scores for Principal Investigator (PI) and Co-principal Investigator (Co-PI) for the Washington University TREC site in 2011, 2013 and 2014

\begin{tabular}{lcccccccc}
\hline & \multicolumn{3}{c}{ PI } & & \multicolumn{3}{c}{ Co-PI } \\
\cline { 2 - 5 } \cline { 7 - 8 } Brokerage Relations & 2011 & 2013 & 2014 & & 2011 & 2013 & 2014 \\
\hline 1. Coordinator & 6.06 & 6.3 & 7.2 & & 7.72 & 10.75 & 8.58 \\
2. Consultant & 16.64 & 6.0 & 11.68 & & 3.89 & 25.54 & 11.61 \\
3. Liaison & 24.06 & 9.85 & 14.64 & & 10.08 & 34.88 & 18.62 \\
4. Representative/Gatekeeper & 11.88 & 5.81 & 7.08 & & 9.58 & 18.65 & 15.58 \\
\hline Total & 25.78 & 10.98 & 16.09 & & 12.99 & 37.96 & 23.28 \\
\hline
\end{tabular}

The liaison role's relatively high global score (1.85) in 2013 suggests that actors in the system are more likely to participate in brokerage activities in which all belong to different disciplines. The high liaison score of the global measure is also related to the high liaison roles of the PI and Co-PI (Table 7). The 2014 global brokerage score is statistically significant, especially for representative/gatekeeper brokerage (2.28). From Table 5, we see that the members 1 and 31 contribute to this brokerage role in addition to the PI and Co-PI.

Figure 2 portrays the Washington University TREC site network, with nodes sized according to total brokerage score and colored according to disciplinary category. In 2011, the principal investigator (PI), an epidemiologist, and the co-principal investigator (Co-PI), a social scientist, were the two primary brokers in the Washington University TREC network. By 2013, more brokerage relations are observed among other network members. Table 5 lists broker relations for each year according to a criterion of $>1.64$. For example, member 1 acted as a coordinator and representative/gatekeeper for the discipline of Medicine in 2013. In 2014, this brokerage role is more solidified for some members: member 1 remains as a representative/gatekeeper, member 28 is a consultant and liaison, and member 31 is a coordinator and representative/gatekeeper.

Standardized brokerage scores for the PI and CoPI for all brokerage types can be found in Table 7. In general, both the PI and Co-PI have higher brokerage scores than other network members across all four brokerage types, indicating that their intermediary roles as a coordinator, consultant, liaison and representative/gatekeeper are all crucial in this network. Their roles as liaisons are especially salient in the network, linking all disciplinary categories. It is also the case that the broker relations of the PI and Co-PI changed through the years. The Co-PI's broker relations were substantially higher in 2013 than 2011, as seen by the higher total brokerage score for the latter year (see Table 7), and decreased 

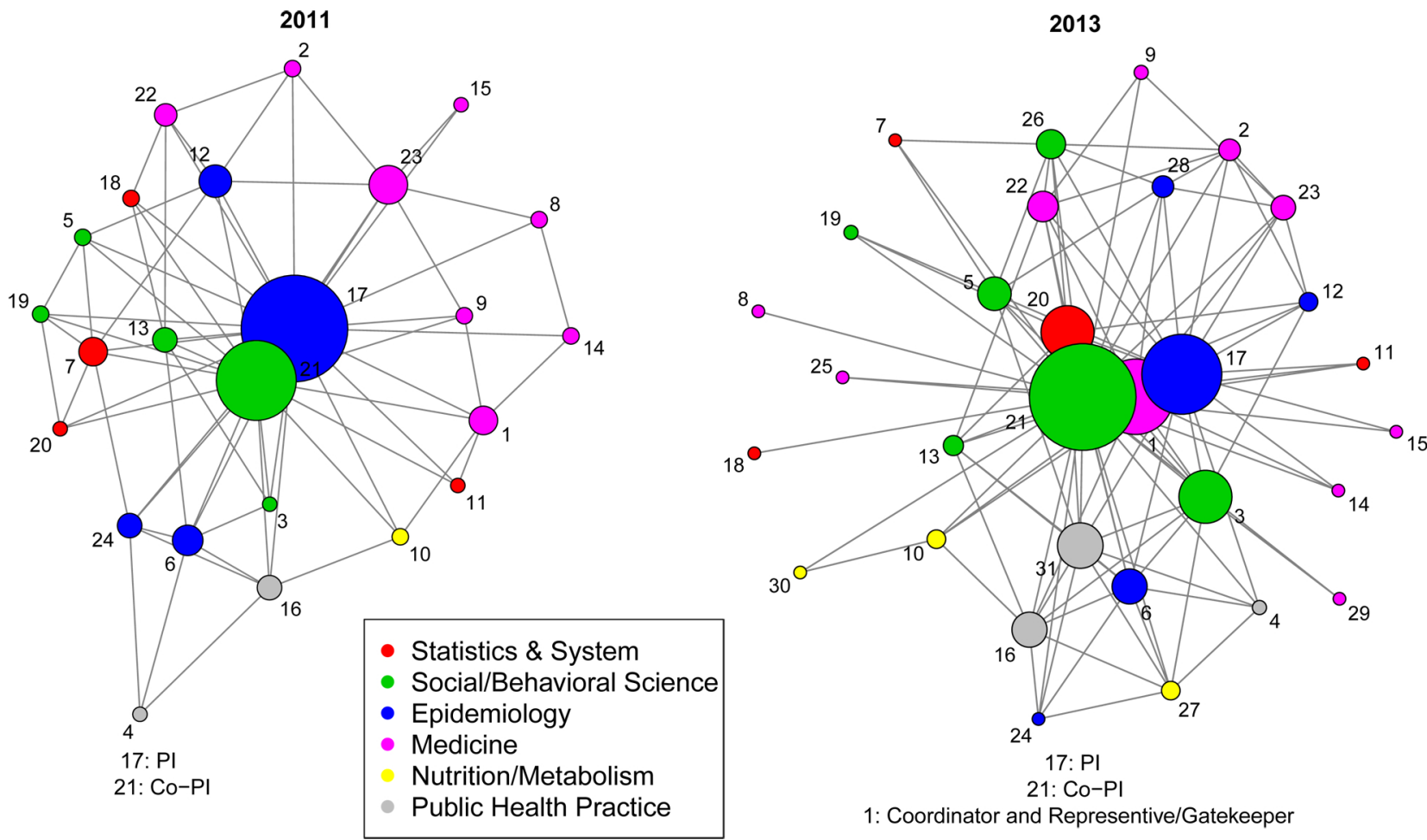

2014

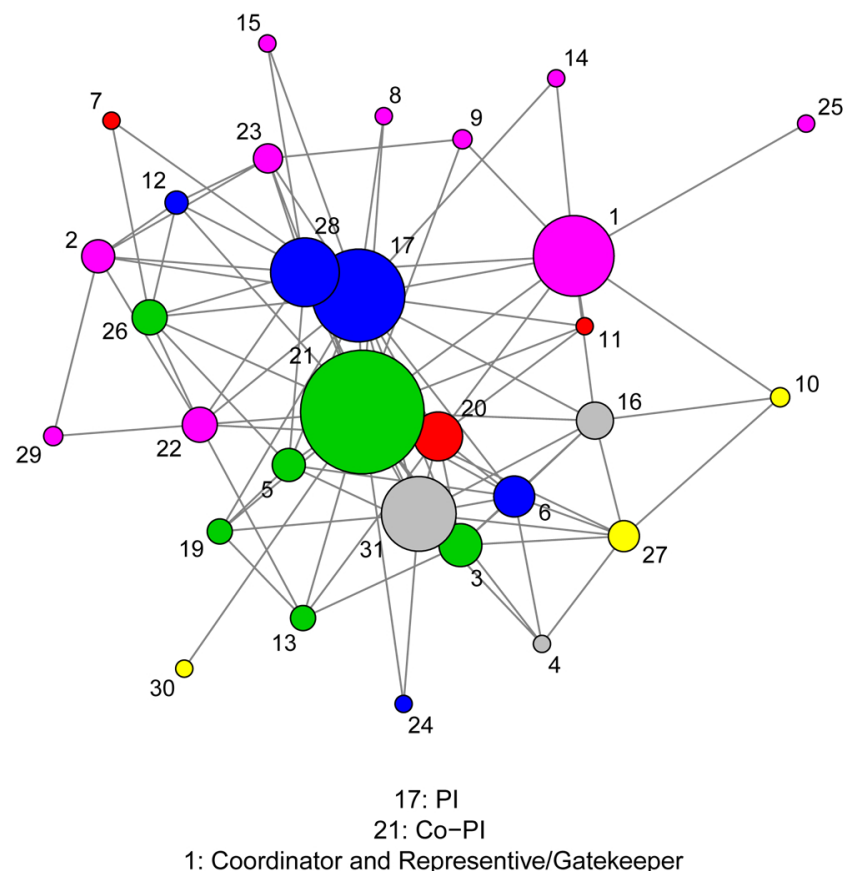

Figure 2: Network Plot of the Washington University TREC site in 2011, 2013 and 2014. The number in nodes is the unique id of investigators, and the node size indicates the total brokerage score of the investigator. The nodes are colored by different disciplines. The nodes of 17 and 21 are downsized than actual brokerage score for visual convenience. In 2011, PI and Co-PI are the main brokers in this network. In 2013 and 2014, more brokerage relations exist (see also Table 5). 
a bit in 2014. Meanwhile, the PI tended to distribute his brokerage role to other members by $2013-14$, evidenced by a lower total brokerage score in 2013-14 than 2011 and the increased numbers of brokers in 2013-14 (see Table 5). It is worth noting that a decrease in brokerage score does not necessarily mean a decrease in collaborative relations. It also can indicate an increase in triangle relations (complete transitivity).

Another natural way to measure brokerage is through the centrality measure known as betweenness $[11], g(w)=\frac{\sum_{s \neq w \neq t} \sigma_{s t}(w)}{\sigma_{s t}}$, where $\sigma_{s t}(w)$ is the number of "paths" from node $s$ to node $t$ that pass through node $w$ and $\sigma_{s t}$ is the number of shortest paths between them. We computed the betweenness for all investigators and found that the correlation between the betweenness and the brokerage score was extremely high (0.99) in all years. In fact, betweenness and brokerage scores are similar in their abilities to measure the extent to which each actor controls the network as a broker. The difference is that betweenness is based on counting the shortest paths of a pair $(i, k)$ that the actor $j$ lies on, which can include more than two-steps relations, whereas the total brokerage score only regards a two steps brokerage process. Thus it is not surprising two measures are empirically close in a small network like ours. We include both, with the knowledge that the brokerage score provides a more precise measure of the kinds of brokerage and clarifies the characteristics that each actor may perform.

Representative brokers are in a better position to link people who might otherwise not link with one another as a result of being in the same discipline. These individuals may therefore be more translational in their research. It is interesting to note that in all years, network investigators from the medical discipline on the whole had the fewest network ties Yet, when the representative brokerages were considered, it became clear that one network member from medicine linked the medical investigators to other members of the network. Coordinators, who broker relationships in which all members are from the same discipline, arguably may be seen as less translational. We intend to follow this pattern over time to see if it is replicated.

\section{Discussion}

A number of findings from our use of social network analysis to assess and provide actionable feedback on the functioning of the Washington University TREC team suggest ways in which the transdisciplinary functioning of the group can be improved. Understanding the patterns and types of brokers within the TREC social network allows plans to be developed for increasing the connectedness of investigators, especially those who have not been well integrated into the network. This raises the likelihood that their perspectives will add to the team's ability to capture the complexity of energy balance and cancer.

Identifying who serves as liaison brokers suggests ways of fostering engagement through communication and training. Likewise, identifying consultant brokers suggests a way of linking members of the same discipline within the network who previously were unlinked. Information on coordinator brokers suggests another way of organizing disciplinary subnetworks within the network, with the goal of better integrating them into the network. Similarly, identifying representative brokers provides a first step toward connecting disciplinary sub-networks of disciplines with the network as a whole.

We found that investigators from the medical discipline had the fewest ties to the TREC network, which was rendered less worrisome when we determined that they were being linked to the network through one network member from medicine who leads one the TREC's four research projects (see Figure 2). This investigator plays a valuable role in transmitting information and ideas between medical discipline investigators and other network members.

We also found that although the PI initially fulfilled the most brokerage functions, by the second time of data collection, he had begun to share these functions with the Co-PI and other network members, thus minimizing burnout and helping to ensure the flow of communication among TREC site investigators. That the two administrators of the site held complementary roles in terms of integrating investigators within the site maximized communication and helped to ensure that information was flowing, thus optimizing the development of new research questions, methods, and analyses that mark the success of transdisciplinary research. 


\subsection{Implications for Process and Training}

The pattern of brokerage across the site suggests areas of focus to ensure optimal transdisciplinary communication while conserving valuable resources, not the least of which is investigator time. This occurs by virtue of providing information to help administrators determine the frequency and structure of team meetings in a way that balances the benefits of the exposure to ideas from the full range of disciplines with the cost of scheduling and implementing meetings. For example, we now know the benefit of including our representative broker from medicine in wider team meetings, because her ties to other network members from medicine will help to ensure that they receive information from other disciplines

Likewise, analysis of brokerages suggests how to optimize team functioning by training investigators to assume certain broker roles. In our example, medical investigator team members were less likely to be engaged with members from other disciplines, perhaps because of their clinical duties and physical location at sites outside the medical campus. Our social network analysis let us realize the importance and viability of having a medical investigator serve as a representative broker role. Had one not been available to serve the role, it would have been prudent to train someone to assume that position. Other brokers, such as liaison brokers, connect investigators from outside their own discipline with members from other disciplines, in other words, they foster transdisciplinary groupings of investigators. This is essential for achieving the benefits of transdisciplinary research. Network analysis allows a team to determine whether the broker function is being filled and if so, how successfully. If neither administrators nor other investigators are assuming the role, a member or members can be trained in the liaison broker role.

We report only for the Washington University TREC in the present paper, in order to investigate one network in depth. We will report on data from all five TREC sites (i.e., the University of Pennsylvania, the University of California-San Diego, Washington University, Harvard University, and the Coordination Center at the Fred Hutchinson Cancer Research Center) for the years reported in the present paper and for 2015. It is possible that the picture seen for Washington University will change when data from other sites are included and another year of data is added.

\section{Conclusions}

Transdisciplinarity and team science have increasingly been recognized for their benefit in advancing new scientific discoveries and moving those discoveries to translation into patient care and community health. Yet, how to achieve the benefits of transdisciplinary research is neither intuitive nor based upon clear guidelines from funders. Social network analysis that considers broker functions on research teams provides a means of assessing team functioning so that changes can be implemented to maximize functioning. These changes, based on the flow of information, allow mid-course corrections to be made rather than second guessing when teams are not functioning as planned.

\section{Acknowledgments}

This research was supported by an NCI grant U54 CA155496.

\section{References}

[1] Rosenfield PL., 1992. The potential of transdisciplinary research for sustaining and extending linkages between the health and social sciences. Soc Sci Med., 35, pp.1343-57.

[2] Hall K, Vogel A, Stipelman B, Stokols D, Morgan G, Gehlert S., 2012. A four-phase model of transdisciplinary research: Goals, team processes, and strategies. Transl Behav Med., 2(4), pp. 415-430.

[3] Emmons KM, Viswanath K, Colditz G., 2008. The role of transdisciplinary collaboration in translating and disseminating health research: lessons learned and exemplars of success. Am J Prev Med., 35(2S), pp. S204-S210.

[4] Patterson RE, Colditz GA, Hu FB, et al., 2013. The 2011-2016 Transdisciplinary Research in Energetics and Cancer (TREC) initiative: rationale and design. Cancer Causes Control, 24(4), pp. 695-704.

[5] Gehlert S, Hall K, Vogel A, et al., 2014. Advancing transdisciplinary research: The Transdisciplinary Energetics and Cancer Initiative. J Transl Med Epidemiol, 2(2), p. 1033.

[6] Aboelela SW, Merrill JA, Carley KM, Larsen E., 2007. Social network analysis to identify an interdisciplinary research center. J Res Admin, 38(1), pp. 61-78. 
Sarah Gehlert, Bobbi J. Carothers, Jung Ae Lee, Jefferson Gill, Douglas Luke, Graham Colditz

A Social Network Analysis Approach to Diagnosing and Improving the Functioning of Transdisciplinary Teams in Public

[7] Wuchty S, Jones BF, Uzzi B, 2007. The increasing dominance of teams in production of knowledge. Science, 316(5827), pp. 1036-1039.

[8] Gould RV, Fernandez RM., 1989. Structures of mediation: a formal approach to brokerage in transaction networks. Sociol Methodol, 19, pp. 89-126.

[9] Spiro ES, Acton RM, Butts CT., 2013. Extended structures of mediation: Re-examining brokerage in dynamic network. Soc Networks, 35, pp. 130-143

[10] Butts CT., 2014. sna: Tools for social network analysis. R package version 2.3-2. 2014; http://CRAN.Rproject.org $/$ package $=$ sna

[11] Freeman LC., 1979. Centrality in social networks: conceptual clarification. Soc Networks, 1, pp. 215239.

\section{About the Authors}

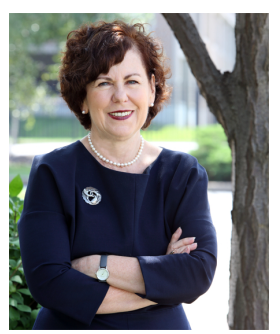

Sarah Gehlert, PhDis the E. Desmond Lee Professor of Racial and Ethnic Diversity at the George Warren Brown School of Social Work and Professor in the Department of Surgery at Washington University in St. Louis. Dr. Gehlert is the Associate Director of the U54 Washington University Transdisciplinary Research in Energetics and Cancer and the Director of its Education, Training, and Outreach Core. She is Co-Director of the Prevention \& Control Program at Washington University in St. Louis. From 2003 to 2009, she was Principal Investigator and Director of the P50 Center for Interdisciplinary Health Disparities Research at the University of Chicago.

Bobbi J. Carothers, PhD is a Senior Data Analyst for the Center for Public Health Systems Science at Washington University in St. Louis. Her current responsibility is to serve as the data analyst for several evaluations: Washington University's Institute of Clinical and Translational Sciences and the Washington University Transdisciplinary Research on Energetics and Cancer. She has served as Adjunct Faculty for the George Warren Brown School of Social Work teaching Applied Linear Modeling.

Jung Ae Lee, PhD is a Postdoctoral Research Associate in the Division of Public Health Sciences, Department of Surgery at Washington University School of Medicine in St. Louis and also a core statistician in the Washington University Transdisciplinary Research on Energetics and Cancer (TREC). Dr. Lee's previous research was built upon statistical methodology, dealing with sample integrity problems in high dimensional low sample size data, such as missing data, batch biases and outliers. As a member of Washington University TREC, Dr. Lee's research interests lie in integrating bioinformatic tools into translational health research, including cancer and chronic disease control.

Jefferson Gill, PhD is a Professor in the Departments of Surgery, Biostatistics and Political Science, and CoInvestigator/Core Leader for the Bioinformatics Core for the Washington University Transdisciplinary Research on Energetics and Cancer. He has done extensive work in the development of Bayesian hierarchical models, nonparametric Bayesian models, elicited prior development from expert interviews, as well in fundamental issues in statistical inference. He has extensive expertise in statistical computing, Markov Chain Monte Carlo (MCMC) tools in particular. Current applied work includes: energetics and cancer, long-term mental health outcomes from children's exposure to war, pediatric head trauma, analysis of mouse models, and molecular models of sickle cell disease.

Douglas Luke, $\mathbf{P h D}$ is Professor and Director of the Center for Public Health Systems Science at the George Warren Brown School of Social Work. Dr. Luke directs work focused primarily on the evaluation, dissemination, and implementation of evidence-based public health policies. In addition to his appointment at the George Warren Brown School of Social Work, Dr. Luke is a member of the Institute for Public Health and a founding member of the Washington University Network of Dissemination and Implementation Researchers.

Graham Colditz, MD, PhD is the Neiss-Gain Professor in the School of Medicine, Chief of the Division of Public Health Sciences in the Department of Surgery, and the Associate Director of Prevention \& Control at the Siteman Cancer Center at Washington University in St. Louis. He is also serving as the deputy director of the Institute for Public Health at Washington University. Dr. Colditz is the principal investigator for Washington University Transdisciplinary Research on Energetics and Cancer. He is also the principal investigator of the Siteman Cancer Center's Program for the Elimination of Cancer Disparities.

Copyright (c) 2015 by the authors. This is an open access article distributed under the Creative Commons Attribution License (https://creativecommons.org/licenses/by/4.0/), which permits unrestricted use, distribution, and reproduction in any medium, provided the original work is properly cited. 